\title{
ВПЕРВЫЕ ДИАГНОСТИРОВАННАЯ ФИБРИЛЛЯЦИЯ ПРЕДСЕРДИЙ И ОЖИРЕНИЕ У ЛЮДЕЙ ПОЖИЛОГО ВОЗРАСТА В ТЕРАПЕВТИЧЕСКОЙ ПРАКТИКЕ
}

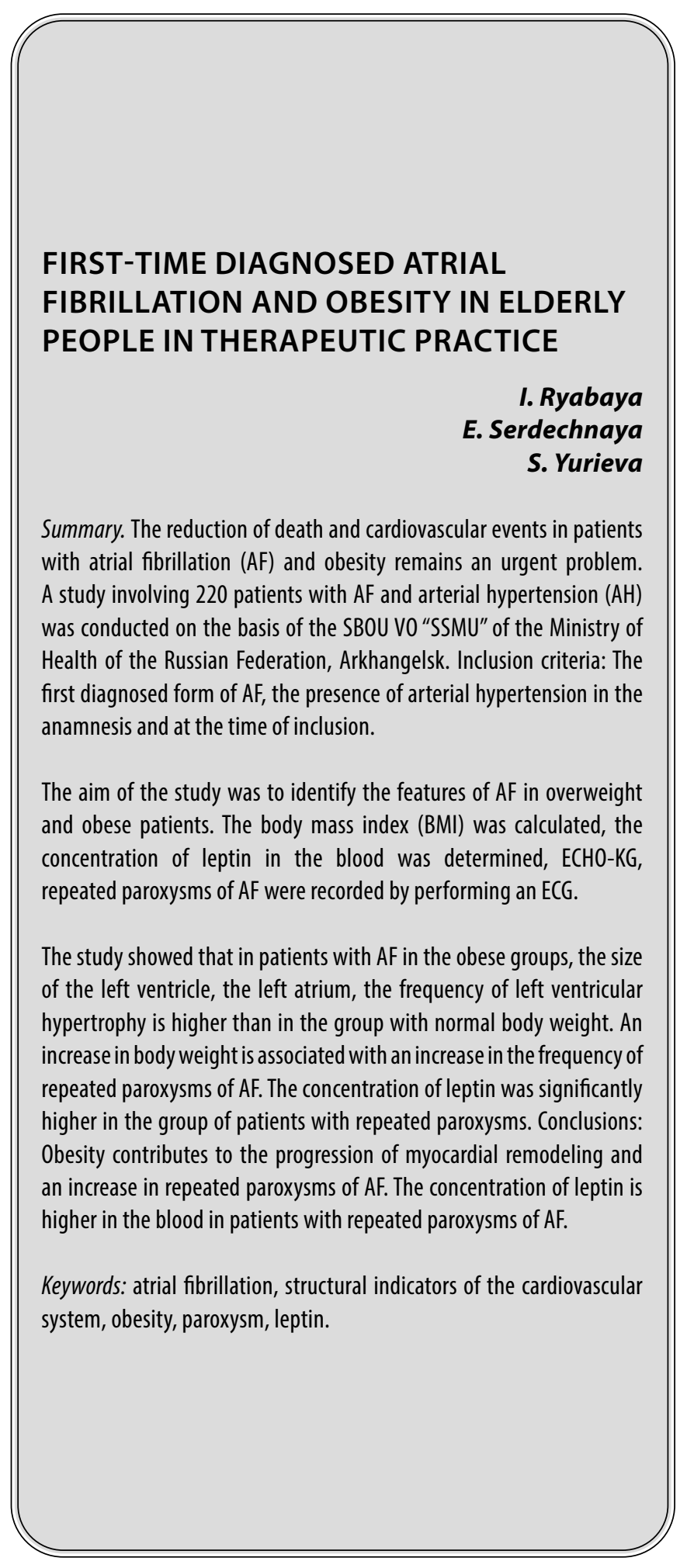

Рябая Ирина Николаевна

К.м.н., Доцент, ФГБОУ ВО «Северный государственный медицинский университет» soldik24@yandex.ru

Сердечная Елена Валерьевна ФГБОУ ВО «Северный государственный медицинский университет»

Д.м.н., Проферессор

Юрьева Светлана Владимировна

К.м.н., Доцент, ФГБОУ ВО «Северный государственный медицинский университет»

Аннотация. Снижение смерти и кардиоваскулярных событий у пациентов с фибрилляцией предсердий (ФП) и ожирением остается актуальной проблемой. На базе ГБОУ ВО «СГМУ» Минздрава России, г. Архангельск проведено исследование с участием 220 пациентов с ФП и артериальной гипертензией (АГ). Критерии включения: Впервые диагностированная форма ФП, наличие артериальной гипертензии в анамнезе и на момент включения.

Цель исследования - выявить особенности ФП у пациентов с избыточной массой тела и ожирением. Выполнен расчет индекса массы тела (ИМТ), определена концентрации лептина в крови, ЭХО-КГ, регистрация повторных пароксизмов ФП осуществлялась посредством выполнения ЭКГ.

Исследование показало, что у пациентов с ФП в группах с ожирением размеры левого желудочка, левого предсердия, частота гипертрофии левого желудочка выше, чем в группе с нормальной массой тела. Увеличение массы тела ассоциируется с повышением частоты повторных пароксизмов ФП. Концентрация лептина достоверно выше в группе пациентов с повторными пароксизмами. Выводы: Ожирение способствует прогрессированию ремоделирования миокарда и увеличению повторных пароксизмов ФП. Концентрация лептина выше в крови у пациентов с повторными пароксизмами ФП.

Ключевые слова: фибрилляция предсердий, структурные показатели сердечно-сосудистой системы, ожирение, пароксизм, лептин. 
$\Phi$ ибрилляция предсердий (ФП) - является одной из самых распространенных аритмий 21 века, ассоциируется с очень высоким риском развития тромбоэмболических осложнений (ТЭО) [8, с. $23 ; 20$, c. 517$]$.

Артериальная гипертензия (АГ) и ожирение значительно повышают риск возникновения и ухудшают течение ФП [3, с. 94; 14, с. 62].

В современном мире, ожирение является серьезной проблемой и широко распространено среди населения. Жировая ткань выступает в роли секреторного органа, способного продуцировать биологически активные вещества - адипокины, влияющие на процессы ремоделирования сердца [13, с. 532].

Проведены немногочисленные исследования, показавшие, что избыточная масса тела и ожирение ухудшают течение ФП [4, 18, с. 70], но также были опубликованы данные, описывающие лучшую выживаемость и меньшую частоту кардиоваскулярных событий у пациентов с ФП, имеющих в анамнезе ожирение [24, с. 34; 17, с. 11]. Учитывая противоречивую информацию, нами было инициировано исследование, целью которого явилось выявить особенности течения ФП у пациентов с избыточной массой тела и ожирением.

Исследование выполнено на кафедре поликлинической терапии и сестринского дела федерального государственного бюджетного образовательного учреждения высшего образования «Северный государственный медицинский университет» Минздрава России, г. Архангельска.

\section{Материалы \\ и мето $\Delta$ ы}

В период с января 2014 года по декабрь 2016 года было обследовано 220 пациентов г. Архангельска. Критерии включения: 1) первый эпизод ФП зафиксирован на электрокардиограмме не ранее 1 года до момента включения в исследование, 2) наличие АГ 1-й - 3-й степени в анамнезе и на момент включения.

Критериями исключения явились: 1) возраст пациента менее 18 лет, 2) постоянная форма ФП, 3) клапанные пороки сердца (ревматические пороки, а также гемодинамически значимые атеросклеротические клапанные пороки), гипертиреоз, синдром WPW.

Проведено первичное обследование пациентов, изучен анамнез ФП, выполнен расчет ИМТ, в начале исследования определен уровень лептина крови методом иммуноферментного анализа.
Всем пациентам проводилось электрокардиографическое исследование (ЭКГ).

Выполнен расчет индекса массы тела: ИМТ (кг/м2) =вес (кг)/рост (м2). На основании ИМТ были выделены четыре группы пациентов: 1-я группа - ИМТ до 25 кг/ M2 (нормальная масса тела) - 42 человек (19,09\%), 2-я группа - ИМТ $25 \leq$ ИМТ < 30 кг/м2 (избыточная масса тела) - 90 человек (40,9\%), 3-я группа - $30 \leq$ ИМТ < 35 кг/м2 (первая степень ожирения) - 59 человек (26,81\%), 4-я группа - ИМТ $\geq 35$ кг/м2 (вторая-третья степень ожирения) - 29 человек (13,2\%).

Эхокардиографическое исследование (ЭХО-КГ) выполнено при включении в исследование и через 1 год с изучением следующих параметров: конечно-диастолический размер левого предсердия (КДР ЛП), конечно-систолический размер левого желудочка (КСР ЛЖ), конечно-диастолический размер левого желудочка (КДР ЛЖ), фракция выброса (ФВ). Определялся индекс массы миокарда левого желудочка (ИММ ЛЖ). Нормальный показатель ИММ Лж $\leq 115$ г/м2-у мужчин и $\leq 95$ г/м2- у женщин [10, с. 755].

Выполнено обучение пациентов контролю пульса на лучевой артерии, а также предоставлялась информация о симптомах, характерных для ФП. Пароксизмы ФП регистрировались при проведении ЭКГ в случае возникновения симптомов, характерных для ФП. Бессимптомные формы выявлялись при ежедневном контроле регулярности пульса на лучевой артерии дважды в сутки (утро, вечер), затем осуществлялось проведение ЭКГ для подтверждения ФП.

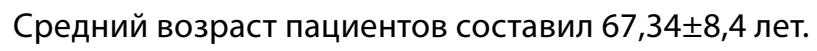

Из анамнеза известно, что до установления диагноза ФП группы пациентов не различались по стадии гипертонической болезни (ГБ). Распределение по стадиям ГБ: ГБ І имели 3 (1,3\%) пациента, ГБ ІІ - 23 (10,5\%), ГБ ІІІ - 194 (88,2\%). Изолированную систолическую артериальную гипертензию имели 21 человек (9,5\%).

С целью исключения патологии щитовидной железы всем пациентам выполнено исследование содержания тиреоидных гормонов в крови (ТТГ, Т3, Т4), ультразвуковое исследование щитовидной железы.

Все пациенты в исследовании имели АГ в анамнезе и на момент включения в исследование. Для лечения АГ использовались следующие схемы:

1. иАПФ/сартан+бета-блокатор+амлодипин

2. иАПФ/сартан+бета-блокатор+диуретик

3. бета-блокатор+сартан/иАПФ

4. иАПФ/сартан+диуретик 
5. иАПФ/сартан+амлодипин

6. иАПФ/сартан+амлодипин+диуретик

7. иАПФ/сартан

Антиаритмическая терапия, используемая у пациентов: 1) Амиодарон, 2) соталол 3) бета-блокатор, 4) комбинация бета-блокатор + амиодарон.

Приверженность терапии составила около 70\%.

Длительность наблюдения за пациентами составила 1 год.

Клиническое исследование зарегистрировано в Единая государственная информационная система учета научно-исследовательских, опытно-конструкторских и технологических работ гражданского назначения» (ЕГИСУ НИОКР) № 01201465727.

Статистическая обработка данных выполнена при помощи пакетов прикладных программ IBM SPSS for Windows (версия 24.0), разработчик - ІВМ, США. Объем выборки определен с помощью программы Epilnfo. С целью описания переменных и сравнительного анализа использовались непараметрические методы описательной статистики, распределение количественных данных проверено на нормальность с помощью теста Шапиро-Уилка. Для описания ненормально распределенных данных использовались медиана и показаны $25 \%-75 \%$ перцентиль, часть данных предствлены в формате $\mathrm{M} \pm \mathrm{SD}$. Сравнения трех групп по количественным признакам проводились на основе непараметрического критерия Краскелла-Уоллеса. Сравнения двух групп по количественным признакам проводились на основе непараметрического критерия Манна-Уитни. Проведены тесты на соблюдение условий, необходимых для применения вышеуказанных методов. Уровень статистической значимости был зафиксирован на уровне вероятности а-ошибки 5\%. Влияния схем лечения артериальной гипертензии на снижение развития повторных пароксизмов ФП было оценено с применением метода множественной логистической регрессии.

При определении предикторов повторных пароксизмов ФП проведен множественный регрессионный анализ с одновременным включением всех потенциальных предикторов в модель. Относительный риск рецидива оценен с помощью отношения шансов.

\section{Результаты исслеАования}

В исследование включены 220 пациентов: мужчины - 73 (33,2\%), женщины - 147 (66,8\%). Среднее время наблюдения - 13,2 месяца.
При включении в исследование все пациенты 220 (100\%) имели впервые диагностированную ФП. 220 человек (100\%) имели в анамнезе артериальную гипертензию, хроническая сердечная недостаточность была диагностирована у 198 (90\%) пациентов, ИБС имели 115 $(52,3 \%)$ пациентов, в том числе перенесенный инфаркт миокарда - 23 (10,5\%), сахарный диабет диагностирован у 22 (10\%) пациентов. По наличию сопутствующих заболеваний в группах с различным показателем ИМТ, группы были сопоставимы.

Проведено сравнение показателей КДР ЛЖ, КСР ЛЖ, ФВ, КДР ЛП, выявлена ГЛЖ в группах пациентов с нормальной массой тела, с избыточной массой тела, с ожирением 1-й ст., с ожирением 2-3-й ст.

Результаты, полученные в начале исследования показывают, что группы различались по параметрам КДР лП.

Показатель КДР ЛП в исследуемых группах достоверно выше в группе с избыточной массой тела-40,0 (37,0$43,0)$ мм по сравнению с группой пациентов с нормальной массой тела-39,0 $(36-41,0)$ мм, $(p=0,036)$, в группе с ожирением 1-й ст.-42,0 (38,0-44,0) мм по сравнению с группой пациентов с нормальной массы тела $(p=0,001)$ и в группе с ожирением 2-3-й ст.-42,0 (39,0-45,0) мм по сравнению с группой пациентов с нормальной массой тела $(p=0,013)$. При сравнении группы с ожирением 1-й ст. и группы с ожирением 2-3-й ст. статистически значимых различий не обнаружено.

Анализ структурных изменений через 1 год, показал, что группы стали различаться по большему количеству структурных показателей сердечно-сосудистой системы: КДР ЛЖ, КСР ЛЖ, КДР ЛП (таблица 1). Показатель КДР Лж в группе с ожирением 2-3-й ст. выше, чем в группе пациентов с нормальной массой тела $(p=0,002)$, а также КДР ЛЖ выше в группе пациентов с ожирением 2-3-й ст. по сравнению с избыточной массой тела $(p=0,003)$.

Показатель КСР Лж в группе с ожирением 1-й ст. выше, чем в группе пациентов с нормальной массой тела $(p=0,04)$, а также КСР ЛЖ выше в группе пациентов с ожирением 2-3-й ст. по сравнению с нормальной массой тела $(p=0,005)$ и по сравнению с избыточной массой тела $(\mathrm{p}=0,020)$.

Показатель КДР ЛП в группе с избыточной массой тела выше, чем в группе пациентов с нормальной массой тела ( $p=0,035)$, а также КДР ЛП выше в группе пациентов с ожирением 1-й ст. по сравнению с нормальной массой тела $(p=0,01)$, КДР ЛП достоверно выше в группе с ожирением 2-3-й ст. по сравнению с группой с нормальной массой тела $(p=0,01)$. 
Таблица 1. Структурные показатели сердечно-сосудистой системы в различных группах ИМТ через 1 год наблюдения.

\begin{tabular}{|l|l|l|l|l|}
\multirow{2}{*}{ Показатель } & \multicolumn{4}{|l|}{ Группы ИМТ } \\
\cline { 2 - 5 } & нормальная масса тела & избыточная масса тела & ожирение 1-й ст. & ожирение 2-3-й ст. \\
\hline \multirow{2}{*}{ КДР ЛЖ (мм) } & 48,5 & $\begin{array}{l}49,0 \\
(46,0-54,0)\end{array}$ & $\begin{array}{l}51,0 \\
(49-55,0)\end{array}$ & $\begin{array}{l}53,0 \\
(51,0-56)\end{array}$ \\
\hline \multirow{2}{*}{ КСР ЛЖ (мм) } & $(45,0-52,0)$ & 32,0 & 33,0 & 35,0 \\
& $(31,0$ & $(30,0-35,0)$ & $(32,0-36,0)$ & $(33,0-38,0)$ \\
\hline \multirow{2}{*}{ ФВ (\%) } & $(29,0-34,0)$ & 63,0 & 62,0 & 61,0 \\
\hline \multirow{2}{*}{ КДР ЛП (мм) } & 63,0 & $(58,0-65,0)$ & $(58,0-65,0)$ & $(58,0-63)$ \\
\hline
\end{tabular}

Примечания: признаки представлены как Мe (Q1- Q3).

ИММ Лж у женщин имел следующую тенденцию:

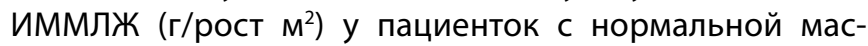

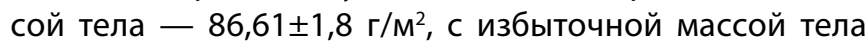

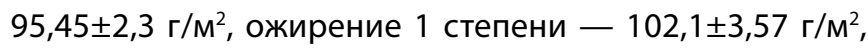
у пациенток со 2-3 степенью ожирения - 112,03 $\pm 4,08$ $\Gamma / \mathrm{M}^{2}$.

У мужчин ИММ Лж в зависимости от группы ИМТ

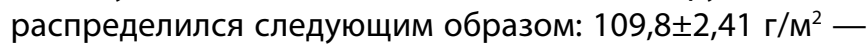
в группе пациентов с нормальной массой тела, $114,63 \pm 2,92$ г/м² - в группе пациентов с избыточной

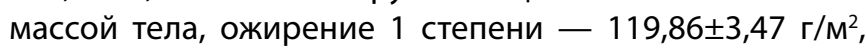

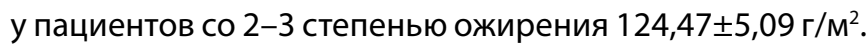

ИММ Лж у женщин имел следующую тенденцию через 1 год наблюдения: ИММЛЖ (г/рост м²) у пациенток

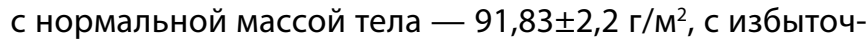

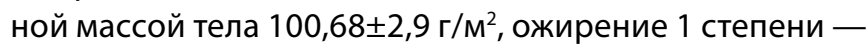
$105,6 \pm 3,63 г / \mathrm{M}^{2}$, упациентоксо 2-3 степеньюожирения$116,28 \pm 4,62 \mathrm{r} / \mathrm{M}^{2}$.

У мужчин ИММ ЛЖ в зависимости от группы ИМТ

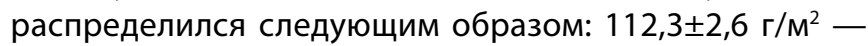
в группе пациентов с нормальной массой тела, $118,51 \pm 3,73$ г/м² - в группе пациентов с избыточной

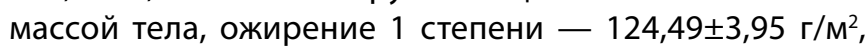

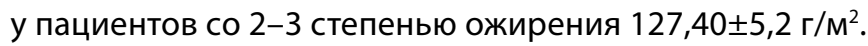

Проведено сравнение частоты повторных пароксизмов ФП в зависимости от наличия избыточной массы тела и ожирения. При оценке рецидивов выявлено: 52 человека $(23,6 \%)$ из 220 не имели повторных пароксизмов. Более половины пациентов - 168 (76,4\%) имели повторные пароксизмы ФП.

Повторные пароксизмы ФП чаще регистрировались у пациентов в группе с ожирением 1-й ст.- $81 \%$ по сравнению с группой с нормальной массой тела $45 \%(p=0,002)$ и у пациентов в группе с ожирением 2-3- й ст.- 90\% по сравнению с группой с нормальной массой тела $-45 \%(p=0,001)$.

Повторные пароксизмы ФП достоверно чаще регистрировались у пациентов из группы с ожирением 1-й ст.- $81 \%$ в сравнении с группой с избыточной массой тела $-66 \%$, $(p=0,003)$ и достоверно чаще регистрировались у пациентов из группы с ожирением 2-3-й ст.- $90 \%$ в сравнении с группой с избыточной массой тела $-66 \%,(p=0,001)$.

Для анализа влияния схем лечения артериальной гипертензии на развитие повторных пароксизмов ФП проведен многофакторный анализ методом множественной логистической регрессии. Статистически значимых взаимосвязей установлено не было.

Выполнен анализ содержания лептина в группах пациентов с повторными пароксизмами ФП и без повторных пароксизмов ФП.

Нами были получены данные, что группы пациентов с повторными пароксизмами ФП в течение 1 года наблюдения и пациентов без повторных пароксизмов ФП различаются по содержанию лептина. Концентрация лептина достоверно выше в группе пациентов с повторными пароксизмами ФП - 20,1 (12,4-32,1) нг/мл, $8,9(7,3-21,6)$ нг/мл - содержание лептина для группы пациентов без повторных пароксизмов ФП ( $p=0,001)$.

Мы выявили предикторы повторных пароксизмов ФП у мужчин и у женщин. Для достижения поставленной цели выполнен многофакторный анализ методом множественной логистической регрессии (методом пошагового отбора независимых переменных). В качестве возможных предикторов рассматривались: концентрация лептина, ИМТ, возраст, КСР ЛЖ, КДР ЛЖ, ФВ, КДР ЛП, ИММ ЛЖ, САД, ДАД, ЧСС, наличие сахарного диабета, инфаркта миокарда, ХСН, ИБС. 
По результатам анализа предикторы повторных пароксизмов ФП у мужчин явились: ИМТ и КДР ЛЖ, у женщин - САД, ИМТ.

\section{Обсужление результатов}

Наше исследование показало, что чем выше ИМТ у пациентов с ФП, тем более выражены структурные изменения (КДР ЛЖ, КСР ЛЖ, ФВ, КДР ЛП, ГЛЖ) через год наблюдения. Эти результаты согласуются с исследованиями, доказывающими, что у пациентов с ожирением происходит увеличение полости левого предсердия и левого желудочка [21, с. 90; 6, с. 35]. Ожирение является фактором риска ГЛЖ [6, с. 36], кроме того, большая группа пациентов в исследовании, особенно с ожирением, не достигли нормотонии, несмотря на проведение антигипертензивной терапии. Ремоделирование миокарда у пациентов с ожирением и избыточной массой тела происходит преимущественно вследствие двух механизмов: за счет синтеза жировой ткани клетками-предшественниками, локализующимися в миокарде, и путем перемещения из эпикардиальной жировой ткани. Жировая ткань затем частично подвергается фиброзу и способствует развитию воспалительных реакций в миокарде с участием макрофагов и провоспалительных цитокинов, приводя к ремоделированию миокарда [23, с. 1294; 15, с. 30].

Частота повторных пароксизмов ФП так же зависит от ИМТ и достоверно выше в группах с ожирением 1-ст. и с ожирением 2-3 ст., чем у пациентов в группах с нормальной и избыточной массой тела. Полученные данные согласуются с результаты немногочисленных исследований подтверждают факт большей распространенности повторных пароксизмов ФП у пациентов с более высоким ИМТ. Вероятнее всего это связано с тем, что ожирение способствует развитию системного воспаления и как следствие фиброзу миокарда, возникает структурное и электрическое ремоделирование миокарда предсердий, что в свою очередь приводит к формированию эктопических фокусов в устьях легочных вен и нарушению нормального электрического проведения по предсердиям [23, с. 1297; 15, с. 32].

У пациентов с повторными пароксизмами ФП значительно выше уровень лептина по сравнению с пациентами без повторных пароксизмов $(p=0,001)$. Данная закономерность может быть связана с профибротическими эффектами лептина, которые были продемонстрированы A. Fukui c коллегами на мышах, а также системным воспалительным влиянием жировой ткани на миокард [9, с. 411], что способствует ремоделированию миокарда и развитию повторных пароксизмов ФП. А.С. Ермаков с соавторами выявили повышенный уровень лептина у женщин с ФП по сравнению с пациентками без ФП, в исследовании участвовали 4937 пациенток, 892 из них имели ФП [7, с. 64].
Нами были выявлены предикторы повторных пароксизмов ФП. У мужчин являлись: ИМТ и КДР ЛЖ, а у женщин - САД, ИМТ. Существующая прямопропорциональная зависимость между величиной ИМТ и ФП доказана на примерах клинических исследований, ожирение влияет на процессы ремоделирования миокарда, что является провоцирующим фактором для развития ФП $[18$, с. $72 ; 22$ с. 2320$]$.

Повышение уровня АД способствует возникновению триггерной активности в предсердиях, развитию структурного и электрического ремоделирования предсердий. Структурное ремоделирование приводит к фиброзу, электрическое ремоделирование вызывает замедление внутрипредсердной проводимости, запускаются реакции системного и локального воспаления. В результате данных процессов формируется односторонний блок проведения возбуждения (re-entry) [2, c; 63; 10, с. 362].

Дедов Д.В. с соавторами в исследовании пациентов с рецидивирующей ФП также установили увеличение КДР ЛЖ у пациентов с рецидивами ФП по сравнению с группой контроля без ФП [1, с. 14]. Ремоделирование миокарда способствует формированию эктопических очагов и нарушению нормального электрического проведения, тем самым способствуя возникновению аритмии [23, с. $1299 ; 15$, с. 35].

\section{Выво $\triangle \mathrm{b}$}

Исследование показало, что у пациентов с фибрилляцией предсердий и наличием ожирения размеры левого желудочка и левого предсердия больше и частота развития ГЛЖ выше, чем у пациентов с нормальной массой тела.

Избыточная масса тела и ожирение способствуют прогрессированию фибрилляции предсердий. Частота повторных пароксизмов значительно увеличивается при повышении индекса массы тела от нормальной массы тела к избыточной массе тела, от избыточной массы тела к ожирению 1-й ст. и 2-3-й ст.

Концентрация лептина достоверно выше у пациентов с повторными пароксизмами фибрилляции предсердий.

Предикторами повторных пароксизмов фибрилляции предсердий у мужчин явились - ИМТ и КДР ЛЖ, у женщин - САД, ИМТ.

Перспективы дальнейшего изучение темы заключаются в более детальной оценке ремоделирования миокарда, в том числе после коррекции избыточной массы тела и ожирения. Также является перспективным направлением изучение влияния других адипокинов на развитие ФП и поиск новых предикторов повторных пароксизмов ФП. 


\section{СоблюАение}

этических станАартов

Дата заседания этического комитета при ГБОУ ВО «Северный государственный медицинский уни- верситет» Минздрава России 18.02.2014, протокол № 01/02-14.

Добровольное согласие на участие в исследовании подписано всеми участниками.

\section{ЛИТЕРАТУРА}

1. Дедов Д.В., Иванов А.П., Эльгардт И.А. Влияние электромеханического ремоделирования сердца на развитие фибрилляции предсердий у больных с ИБС и артериальной гипертонией. Российский кардиологический журнал. 2011.

2. Цыпленкова Н.С., Панова Е.И., Жданкина Н.В., Морозова Е.П., Каратаева О.В. Фибрилляция предсердий у больных с ожирением и артериальной гипертензией. Архивъ внутренней медицины. 2015.

3. Чумакова Г.А., Веселовская Н.Г., Козаренко А.А., Воробьева Ю.В. Особенности морфологии, структуры и функции сердца при ожирении. Российский кардиологический журнал. 2012.

4. Abed H, Wittert G, Leong D, Shirazi M, Bahrami B, Middeldorp M, et al. Effect of Weight Reduction and Cardiometabolic Risk Factor Management on Symptom Burden and Severity in Patients With Atrial Fibrillation. JAMA.2013; 310(19): 2050. doi: 10.1001/jama.2013.280521

5. Andrade J, Khairy P, Dobrev D, Nattel S. The clinical profile and pathophysiology of atrial fibrillation: relationships among clinical features, epidemiology, and mechanisms. Circ. Res.2014;114(9):1453-68. doi: 10.1161/circresaha.114.303211

6. Avelar E, Cloward T, Walker J, Farney R, Strong M, Pendleton R, et al. Left Ventricular Hypertrophy in Severe 0besity. Hypertension.2007; 49(1):34-39. doi:10.1161/01.hyp.0000251711.92482.14

7. Ermakov S, Azarbal F, Stefanick, LaMonte M, Li W, Tharp K, et al. The associations of leptin, adiponectin and resistin with incident atrial fibrillation in women. Heart.2016;102(17):1354-62.doi: 10.1136/heartjnl-2015-308927

8. Ferrari R, Bertini M, Blomstrom-Lundqvist C, Dobrev D, Kirchhof P, Pappone C, et al. An update on atrial fibrillation in 2014: From pathophysiology to treatment. International Journal of Cardiology. 2016;203:22-29.doi: 10.1016/j.ijcard.2015.10.089

9. Fukui A, Takahashi N, Nakada, Masaki T, Kume 0, Shinohara T, et al. Role of Leptin Signaling in the Pathogenesis of Angiotensin II-Mediated Atrial Fibrosis and Fibrillation. Circulation: Arrhythmia and Electrophysiology.2013;6(2):402-409.doi: 10.1161/circep.111.000104

10. Goudis CA, Korantzopoulos P, Ntalas IV, Kallergis E, Ketikoglou D. Obesity and atrial fibrillation: A comprehensive review of the pathophysiological mechanisms and links. J. Cardiol. 2015;66(5):361-369. doi: 10.1016/j.jjcc.2015.04.002

11. Goudis CA, Korantzopoulos P, Ntalas IV, Kallergis, EM, Ketikoglou DG. Obesity and atrial fibrillation: A comprehensive review of the pathophysiological mechanisms and links. J. Cardiol. 2015;66 (5):361-369. doi: 10.1016/j.jjcc.2015.04.002

12. Grundvold I, Bodegard J, Nilsson PM, Svennblad B, Johansson G, Östgren C, et al. Body weight and risk of atrial fibrillation in 7169 patients with newly diagnosed type 2 diabetes; an observational study. Cardiovascular Diabetology. 2015;14:5. doi: 10.1186/s12933-014-0170-3

13. Han S, Quon M, Kim J, Koh K. Adiponectin and Cardiovascular Disease. Journal of the American College of Cardiology. 2007; 49(5):531-538.doi:10.1016/j. jacc.2006.08.061

14. Hohnloser S, Pajitnev D, Pogue J, Healey J, Pfeffer M, Yusuf S, et al. Incidence of Stroke in Paroxysmal Versus Sustained Atrial Fibrillation in Patients Taking Oral Anticoagulation or Combined Antiplatelet Therapy. Journal of the American College of Cardiology. 2007;50(22):2156-61.doi: 10.1016/j.jacc. 2007.07.076

15. Lin Y, Chen Y, Chen S. Potential atrial arrhythmogenicity of adipocytes: Implications for the genesis of atrial fibrillation. Medical Hypotheses. 2010;74(6):102629.doi: 10.1016/j.mehy.2010.01.004

16. Marwick T, Gillebert T, Aurigemma G, Chirinos J, Derumeaux G, Galderisi M, et al. Recommendations on the Use of Echocardiography in Adult Hypertension: A Report from the European Association of Cardiovascular Imaging (EACVI) and the American Society of Echocardiography (ASE). Journal of the American Society of Echocardiography.2015;28(7):727-754.doi: 10.1016/j.ech0.2015.05.002

17. Ogawa H, Hamatani Y, Doi K, Tezuka Y, An Y, Ishii M, et al. Sex-Related Differences in the Clinical Events of Patients With Atrial Fibrillation.The Fushimi AF Registry. Circulation Journal.2017;81(10):1403-10. doi:10.1253/circj.cj-17-0071

18. Pathak R, Middeldorp M, Meredith M, Mehta A, Mahajan R, Wong C, et al. Long-Term Effect of Goal-Directed Weight Management in an Atrial Fibrillation Cohort. Journal of the American College of Cardiology. 2015; 65(20): 2159-69.doi: 10.1016/j.jacc.2015.03.002

19. Pathak RK, Mahajan R, Lau DH, Sanders P. The implications of obesity for cardiac arrhythmia mechanisms and management. Can. J. Cardiol. 2015 ; 31: 203-210. doi: 10.1016/j.cjca.2014.10.027

20. Stewart S. Population prevalence, incidence, and predictors of atrial fibrillation in the Renfrew/Paisley study. Heart. 2001;86(5):516-521. doi: 10.1136/ heart.86.5.516

21. Stritzke J, Markus M, Duderstadt, S, Lieb W, Luchner A, Döring, et al. The Aging Process of the Heart: Obesity Is the Main Risk Factor for Left Atrial Enlargement During Aging. Journal of the American College of Cardiology. 2009; 54(21):1982-89.doi: 10.1016/j.jacc.2009.07.034

22. Tedrow UB, Conen D, Ridker PM, Cook NR, Koplan BA, Manson JE, et al. The long- and short-term impact of elevated body mass index on the risk of new atrial fibrillation the WHS (women's health study). J. Am. Coll. Cardiol. 2010; 55(21): 2319-2327.doi: 10.1016/j.jacc.2010.02.029 
23. Wong C, Ganesan A, Selvanayagam J. Epicardial fat and atrial fibrillation: current evidence, potential mechanisms, clinical implications, and future directions. European Heart Journal. 2016;38:1294-1302.doi:10.1093/eurheartj/ehw045

24. Wyse DG, Waldo AL, DiMarco JP, Domanski MJ, Rosenberg Y, Schron EB, et al. A Comparison of Rate Control and Rhythm Control in Patients with Atrial Fibrillation. New England Journal of Medicine.2002;347(23):1825-33. doi:10.1056/nejmoa021328

(с) Рябая Ирина Николаевна ( soldik24@уаndex.ru),

Сердечная Елена Валерьевна, Юрьева Светлана Владимировна. Журнал «Современная наука: актуальные проблемы теории и практики»

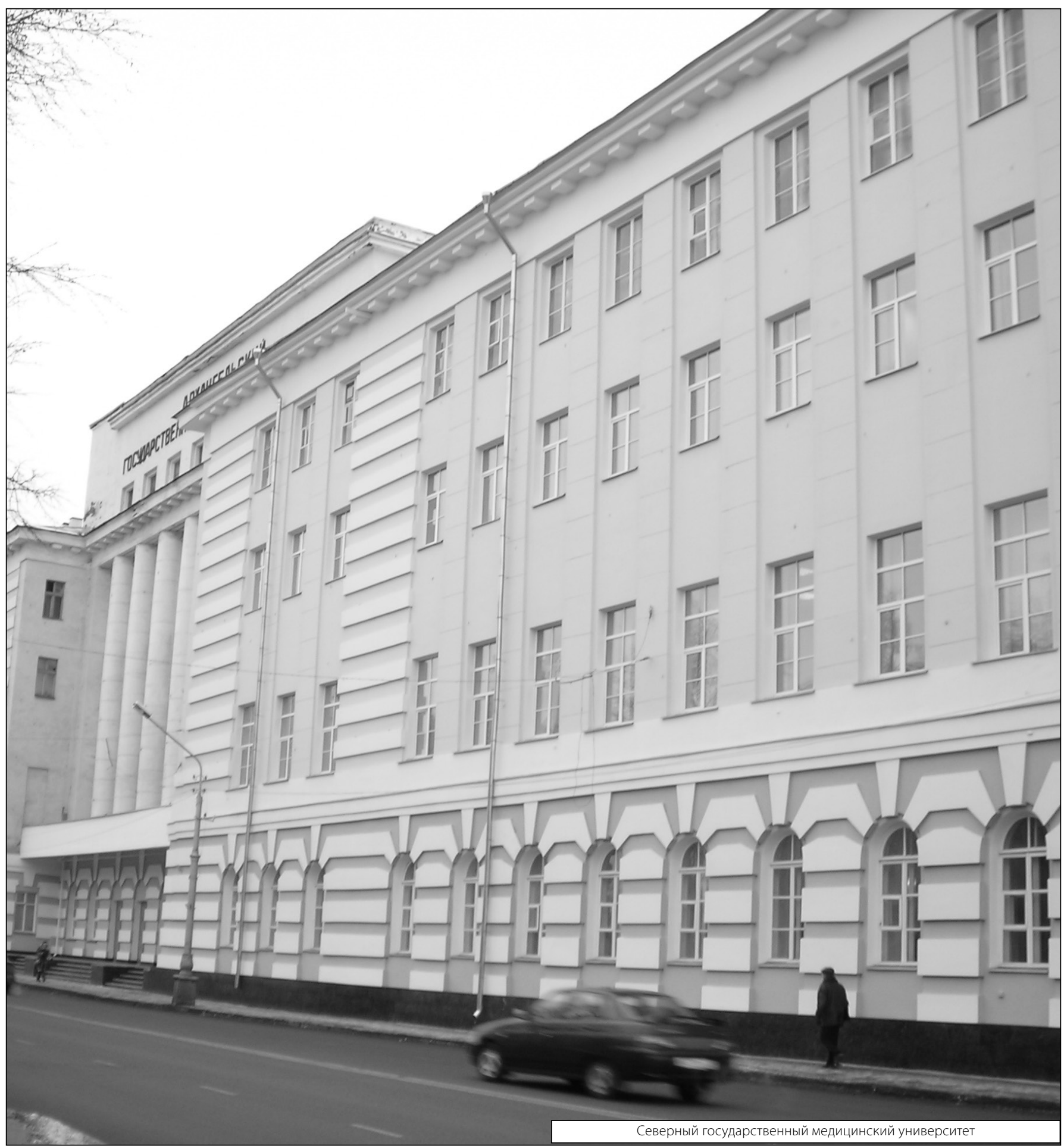

